\author{
M. Scholing \\ T. P. Saltzherr \\ P. H. P. Fung Kon Jin \\ K. J. Ponsen \\ J. B. Reitsma \\ J. S. Lameris \\ J. C. Goslings
}

\section{The value of postmortem computed tomography as an alternative for autopsy in trauma victims: a systematic review}

Received: 25 November 2008

Revised: 1 March 2009

Accepted: 13 April 2009

Published online: 21 May 2009

(C) The Author(s) 2009.

This article is published with open access at Springerlink.com

M. Scholing · T. P. Saltzherr $(\bowtie)$.

P. H. P. Fung Kon Jin - K. J. Ponsen

J. C. Goslings

Academic Medical Center,

Trauma Unit, Department of Surgery, Meibergdreef 9,

1105, AZ,

Amsterdam, The Netherlands

e-mail: t.p.saltzherr@amc.uva.nl

Tel.: +31-20-5666676

Fax: +31-20-6914858

\section{J. B. Reitsma}

Department of Clinical Epidemiology,

Biostatistics and Bioinformatics,

Academic Medical Center,

Amsterdam, The Netherlands

J. S. Lameris

Department of Radiology,

Academic Medical Center,

Amsterdam, The Netherlands
Abstract The aim of this study was to assess the role of postmortem computed tomography (PMCT) as an alternative for autopsy in determining the cause of death and the identification of specific injuries in trauma victims. A systematic review was performed by searching the EMBASE and MEDLINE databases. Articles were eligible if they reported both PMCT as well as autopsy findings and included more than one trauma victim. Two reviewers independently assessed the eligibility and quality of the articles. The outcomes were described in terms of the percentage agreement on causes of death and amount of injuries detected. The data extraction and analysis were performed together. Fifteen studies were included describing 244 victims. The median sample size was 13 (range 5-52). The percentage agreement on the cause of death between PMCT and autopsy varied between 46 and 100\%. The overall amount of injuries detected on
CT ranged from 53 to $100 \%$ compared with autopsy. Several studies suggested that PMCT was capable of identifying injuries not detected during normal autopsy. This systematic review provides inconsistent evidence as to whether PMCT is a reliable alternative for autopsy in trauma victims. PMCT has promising features in postmortem examination suggesting PMCT is a good alternative for a refused autopsy or a good adjunct to autopsy because it detects extra injuries overseen during autopsies. To examine the value of PMCT in trauma victims there is a need for welldesigned and larger prospective studies.

Keywords Review - Postmortem . CT $\cdot$ Autopsy $\cdot$ Trauma
Abbreviations CT: computed tomography - PMCT: postmortem computed tomography $\cdot \mathrm{MRI}$ : magnetic resonance imaging

\section{Introduction}

Trauma is one of the leading causes of death [1]. Injuries cause 5 million deaths every year worldwide, which accounts for $9 \%$ of global mortality. The numbers for morbidity are even higher and injury accounts for $16 \%$ of the global burden of disease [2]. Since the 1980s trauma mortality has been described in epidemiology studies [311]. All of these studies contain valuable information for prevention purposes but also serve as a feedback tool for possible improvements in trauma assessment.
Autopsy is considered the reference standard for postmortem evaluation regarding the detection of the causes of death and sustained (traumatic) injuries. In most cases, autopsy results can explain the cause of death, the number of injuries sustained, and the trauma mechanism in trauma victims. Furthermore clinical autopsies are performed to evaluate potential missed injuries after unsuccessful trauma resuscitation. In most countries, clinical autopsies are performed with permission from the family of the deceased. As a consequence of its invasiveness, permission to conduct a clinical autopsy is often not given. Unlike clinical autopsies, 
forensic (or medicolegal) autopsies may be ordered by the legal authority, which has the sole power to order this type of autopsy. Even though this examination often might seem medically desired, it is not always indicated for medicolegal purposes and is therefore frequently omitted.

In addition, autopsies are very time consuming and labor intensive. The current declining number of autopsies [1215] could mean that valuable data regarding the actual traumatic injuries and causes of death will be either hard to obtain, misclassified, or even lost [16, 17]. To circumvent this problem several institutes are studying the value of postmortem computed tomography (PMCT) in continuation with or without magnetic resonance imaging (MRI) [18-20]. Recently, there have been great improvements in the quality and multiplanar reformatting of CT imaging. Since this radiological technique is noninvasive and an accepted imaging technique its use could be an alternative for clinical autopsy.

The aim of this review was to investigate the value of PMCT as a reliable substitute for autopsy in trauma victims for revealing the cause of death and diagnosing specific injuries.

\section{Material and methods}

A systematic search of the literature was conducted to identify studies examining the value of postmortem computed tomography (PMCT) as an alternative for autopsy to diagnose the cause of death and specific injury in trauma victims.

\section{Criteria for inclusion}

Eligible articles were selected if: (1) the index test(s) included a PMCT, (2) the reference standard(s) included autopsy, (3) the aim of the selected studies was to determine the cause of death and/or major injury, and (4) the included patients were trauma victims. Trauma was defined as a physical injury or wound caused by an external force which may cause death or permanent disability.

\section{Search strategy}

The MEDLINE and EMBASE database were searched with the following free text and MeSH search terms: (autopsy OR necropsy OR obduction OR abduction OR postmortem OR forensic investigation) AND (computed tomography OR CAT scan OR MSCT OR CT) AND (trauma). There was no language, age, or publication year restriction, and articles were included if they evaluated two or more trauma victims.

Additionally, the reference list of each eligible article was screened for other relevant publications (cross- reference search) to identify additional studies not found in the computerized search (MS). Furthermore, a manual search of the following journals was performed (MS) that reported most frequently about the topic of interest: Forensic Science International; Forensic Science, Medicine, and Pathology; Rechtsmedizin; The American Journal of Forensic Medicine and Pathology; International Journal of Legal Medicine; Journal of Forensic and Legal Medicine; Journal of Clinical Forensic Medicine; Legal Medicine (Tokyo); Journal of Trauma; Injury; RöFo; and Radiology.

Meeting abstracts, unpublished data, and theses were not included in our search.

The last search was performed in May 2008.

\section{Selection of dedicated studies}

Two reviewers (MS \& TPS) performed the literature search together. Both reviewers (MS \& TPS) independently assessed the titles of the literature search to determine whether they were potentially relevant. Abstracts of the potentially relevant titles were retrieved. After that, both reviewers assessed the abstracts of the selected titles and retrieved the full text articles of potentially relevant abstracts. Again if there was any doubt if an abstract should be included or the abstract was absent, the full text of the article was retrieved. The final step of inclusion was always based on the full text article. In case of a disagreement the third reviewer (PHPFKJ) was consulted. During the selection process no concealment of authors/ institutions was used.

\section{Assessment of study quality}

Two reviewers (MS \& TPS) independently assessed the quality of the selected studies. We used an adapted version of the QUADAS tool as our quality assessment tool [21]. The QUADAS tool is a validated, generic tool for the assessment of quality in diagnostic accuracy studies. It consists of individual items addressing potential sources of bias and variation in accuracy studies. We selected all relevant items and added additional items specific for this review. Two reviewers independently assessed methodological quality, and, in case of a disagreement, a third reviewer (PHPFKJ) was consulted.

For quality assessment we have analyzed the overall description of the study methods and the final and/or intermediate results reported (i.e., inclusion parameters, study population, the technique of CT employed and autopsies performed, the time period between the examinations, blinding of the examinations, etc.). Studies were considered poor quality and were excluded if they had a lack of information on inclusion criteria, PMCT and/or autopsy description, and had no evidence of blinding. 


\section{Data extraction}

Data were extracted by the two reviewers (MS \& TPS). Because of the complexity of the data, this was performed together. Furthermore, every corresponding author was contacted if the reported data were unclear or incomplete.

\section{Statistical analysis}

The main analysis focused on the proportion of patients in which the cause of death determined by postmortem CT was in agreement with the cause of death established during autopsy (the reference standard). For each study, we calculated this percentage agreement together with the $95 \%$ confidence interval based on the method of Wilson [22]. These individual estimates were then plotted together in a forest plot to give a visual impression of the amount of heterogeneity. A random effects pooling was performed using the logit transformed proportion of agreement as outcome variable, while using the exact binomial distribution to account for differences in precision in estimated proportions across studies. The random effects pooling was performed in SAS using the nonlinear mixed model procedure (SAS version 9.1, SAS Institute Inc., Cary, NC, USA). The $I^{2}$ value was calculated as a measure of inconsistency, where the $I^{2}$ describes the percentage of total variation across the studies that is due to heterogeneity rather than chance [23]. Similar analyses were done for the percentage agreement between $\mathrm{CT}$ and autopsy for specific types of injuries. Again the findings during autopsy were considered as the correct ones (reference standard).

\section{Results}

Search strategy and selection

The computerized search resulted in 465 titles from the EMBASE database and 849 titles from the MEDLINE database (Fig. 1). After reviewing the titles and eliminating the duplicates from both databases 71 titles were selected for further evaluation. Based on the abstracts, 32 papers were excluded because they did not match the inclusion criteria resulting in 39 full text articles that were reviewed. Based on the full text 23 articles of the 39 (59\%) were not eligible for quality assessment because they did not meet our inclusion criteria (Fig. 1). The manual search and crossreference search resulted in three additional papers. In total 19 papers were eligible for quality assessment [24-42]. Within these 19 papers four articles were excluded: one because the descriptive data were not suitable for data extraction and the author could not be contacted, i.e., the data were merely descriptive and no numerical data could be obtained [40]; the other three studies were excluded because of poor quality $[39,41,42]$. The Cohen's kappa coefficient for full text selection was 0.79 which can be considered to indicate substantial agreement [43].

All data were extracted from the remaining 15 studies (Table 1). All 15 studies were observational studies, 8 of them had retrospective data collection $(53 \%)$ and 7 prospective (46\%). Twelve studies provided data about the percentage agreement on the causes of death and seven studies reported specific data about the injuries per anatomical region.

The flowchart of the included studies is represented in Fig. 1 [44].

\section{Data extraction}

Data were extracted from all 15 studies [24-38]. We contacted the authors of 6 studies because data were insufficient or incomplete and received the requested data. The 15 studies included a total of 244 patients with a median sample size of 13 (range 5-52).

The period between the time of death and CT varied from 3 to $192 \mathrm{~h}$, and the time between death and autopsy varies from 15 to $240 \mathrm{~h}$. Standard autopsy implied dissection of the chest, abdomen, and pelvis with all internal organs being examined. Neuropathological examination implied an accurate dissection of the cranium and neck. Standard CT was performed with an MSCT system with which the skull was imaged to the pelvis, the distal femur or the toes with multiplanar reconstructions. However, the protocols for CT, when described, varied widely regarding the type of MSCT machines (2- to 16-slice CT systems). The slice thickness varied between 1.25 and $5 \mathrm{~mm}$ for the head and neck, and 1.25 and $10 \mathrm{~mm}$ for the torso. Furthermore, multiple variations were found in rotation and table speeds, strength of radiation used, and the usage of and methods for multiplanar reconstructions.

Table 2 shows the data of the twelve studies about trauma mechanisms and the percentage agreement on the causes of death found by PMCT and autopsy.

Figure 2 shows a forest plot of the 11 studies which had numerical data on the causes death found by PMCT and autopsy. Four studies had numerical data on specific injuries diagnosed with CT but not on the causes of death. These four studies were therefore omitted in Fig. 2. This plot also gives information about the heterogeneity of the data with an inconsistency $I^{2}$ of $76.4 \%$.

Table 3 shows the results of the seven studies reporting data about the injuries detected by PMCT and autopsy. We have clustered the injuries per body region as described in the abbreviated injury scale (AIS) [45], while the injury of the face was added to the head/neck region. One of the reasons for using this classification is that different levels of injury severity can be clearly defined (e.g., from skin contusion to severe liver laceration). Another reason is that several trauma studies only report on injuries sustained in specific regions. For the face/head/neck region PMCT found 


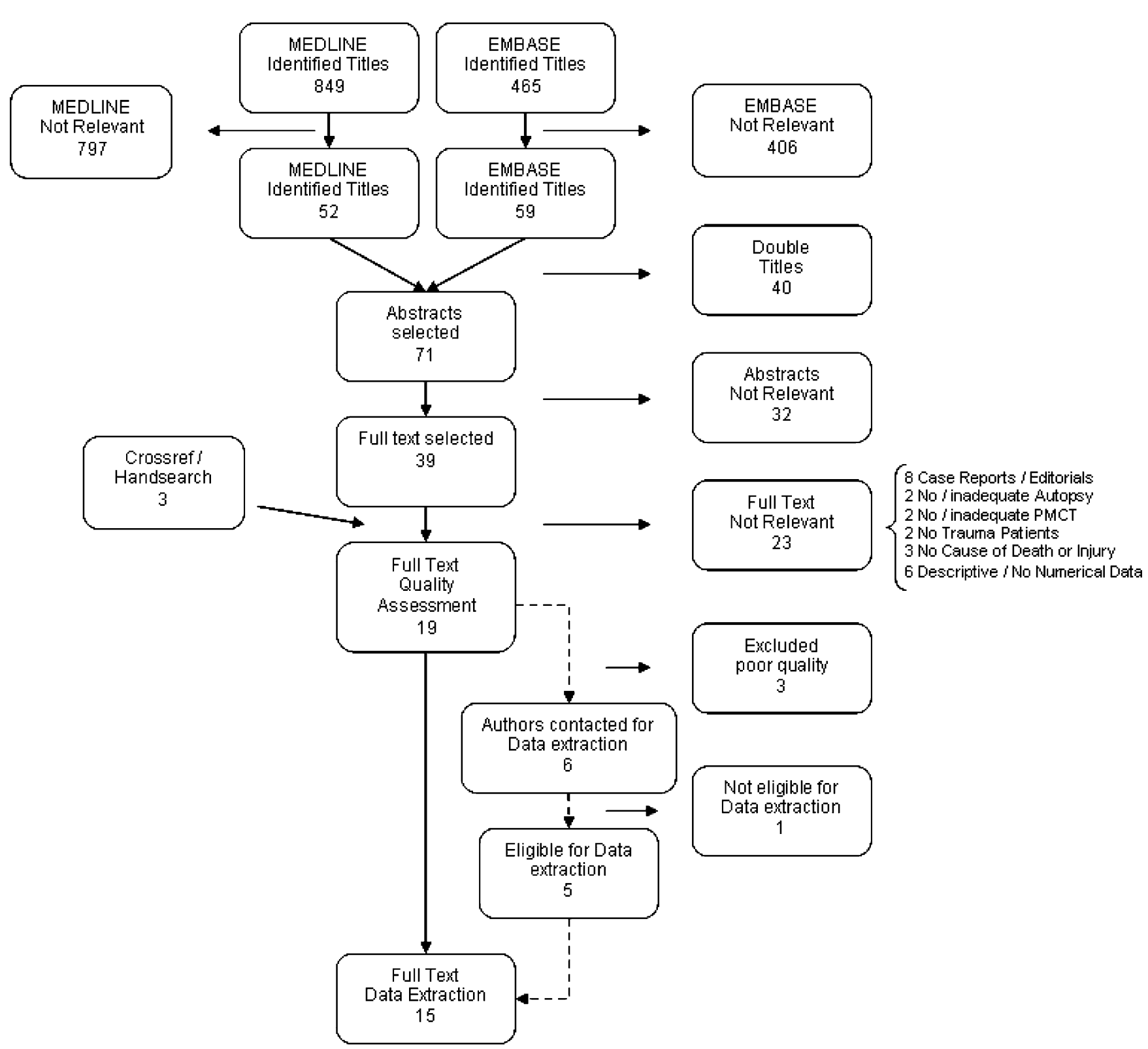

Fig. 1 QUOROM flowchart

between 53 and $100 \%$ of the injuries, and in particular the gunshot injuries were very accurate. The chest region had an even higher percentage of detected injuries $(75-100 \%)$. Although the percentage of detected injuries of the abdomen is high, the absolute number of injuries found in this body region was limited, with Hoey et al. reporting the highest number, 10 for PMCT out of 13 injuries detected at autopsy [29]. One study is missing in Table 3 because the reference standard was a combination of the findings on autopsy and PMCT [32]. In this specific study, the PMCT detected 15\% of the total number of injuries that were missed during autopsy. As a result the percentage agreement between PMCT and autopsy was not $100 \%$ but $85 \%$.

\section{Discussion}

The percentage agreement on the cause of death found by PMCT and autopsy in this study ranged from 46 to $100 \%$. For all the body-region-specific injuries the percentage agreement also varied considerably between 53 and $100 \%$. Autopsies have been performed for centuries, whereas PMCT has been used as a postmortem examination method for no longer than a decade. The far greater experience with autopsies could explain some of the differences in reported percentage agreement between PMCT and autopsy. Because we expect that interpretation of PMCT is subject to a learning curve, the differences will probably decrease 
Table 1 Study characteristics of the 15 eligible studies

\begin{tabular}{|c|c|c|c|c|c|}
\hline First author and ref. no. & Country & Journal & Year & Language & Eligible trauma patients $(n)$ \\
\hline Thali [35] & Switzerland & J. Forensic Sci. & 2003 & English & 33 \\
\hline Yen $[38]^{\mathrm{a}}$ & Switzerland & Forensic Sci. Int. & 2007 & English & 52 \\
\hline Paperno [33] & Germany & $R \ddot{o} F_{O}$ & 2005 & German & 11 \\
\hline Levy [31] & USA & Radiology & 2006 & English & 13 \\
\hline Hoey $[29]^{\mathrm{a}}$ & Israel & J. Trauma & 2007 & English & 12 \\
\hline Yen [36] & Switzerland & J. Magn. Reson. Imaging & 2005 & English & 9 \\
\hline Yen $[37]^{\mathrm{a}}$ & Switzerland & Int. J. Legal Med. & 2005 & English & 5 \\
\hline Christe [26] & Switzerland & Eur. Radiology & 2008 & English & 10 \\
\hline Harcke [28] & USA & Am. J. Forensic Med. Pathol. & 2007 & English & 13 \\
\hline Levy [32] & Israel & Isr. Med. Assoc. J. & 2007 & English & 6 \\
\hline Rutty [34] & UK & J. Forensic Sci. & 2007 & English & 5 \\
\hline Donchin $[27]^{\mathrm{a}}$ & Israel & J. Trauma & 1994 & English & 13 \\
\hline Andematten [25] & Switzerland & Leg. Med. (Tokyo) & 2008 & English & 22 \\
\hline Leth $[30]^{\mathrm{a}}$ & Denmark & Forensic Sci. Med. Pathol. & 2006 & English & 16 \\
\hline Aghayev $[24]^{\mathrm{a}}$ & Switzerland & J. Thorac Imaging & 2008 & English & 24 \\
\hline
\end{tabular}

${ }^{\mathrm{a}}$ Author was contacted

when there is more research performed on and more experience with PMCT. Second, the diversity in the studies' CT protocols, population size, the experience level of imaging interpretation, subject population, and different institutional levels could account for the substantial heterogeneity in the reported percentages of agreement. One of the 15 studies described a study population of more than 40 patients, 4 studies included less than 10 patients, and the median population size was 13. In addition the $\mathrm{CT}$ techniques and the methods for multiplanar reconstructions were different for most studies.
However, according to all protocols the patients were at least examined from skull to the pelvis or for neuroimaging from skull to the first thoracic vertebral body.

Furthermore, the level of injuries described as the cause of death varied from bleeding in the thoracic cavity to hemorrhagic shock from a ruptured thoracic aorta. One study even reported combined causes of death [35]. Therefore autopsy reported more causes of death than patients. Although this can be common in forensic and medical practice, it makes the method of data interpretation and testing more difficult and different from the other tests.

Table 2 Percentage agreement on causes of death

\begin{tabular}{llll}
\hline First author and ref. no. & Trauma mechanism & TP/TP + FN & Agreement on cause of death (\%) \\
\hline Rutty [34] & Various & $5 / 5$ & 100 \\
Paperno [33] & Various & $9 / 11$ & 82 \\
Hoey [29] & Various & $10 / 12$ & 83 \\
Thali [35] & Various & $19 / 41$ & 46 \\
Leth [30] & Various & $14 / 16$ & 88 \\
Harcke [28] & GSW & $13 / 13$ & 100 \\
Levy [31] & GSW & $13 / 13$ & 100 \\
Andematten [25] & GSW & $17 / 22$ & 77 \\
Aghayev [24] & Blunt chest trauma & $-/-$ & 85 \\
Christe [26] & Drowning & $10 / 10$ & 100 \\
Yen [38] & Neurotrauma & $19 / 24$ & 79 \\
Yen [36] & Head + neck trauma & $2 / 4$ & 50 \\
\hline
\end{tabular}

GSW gunshot wounds, TP causes of death diagnosed with PMCT, FN causes of death diagnosed with autopsy and overseen on PMCT, Various mixed trauma mechanisms

${ }^{\mathrm{a}}$ Multiple causes of death per patient possible 


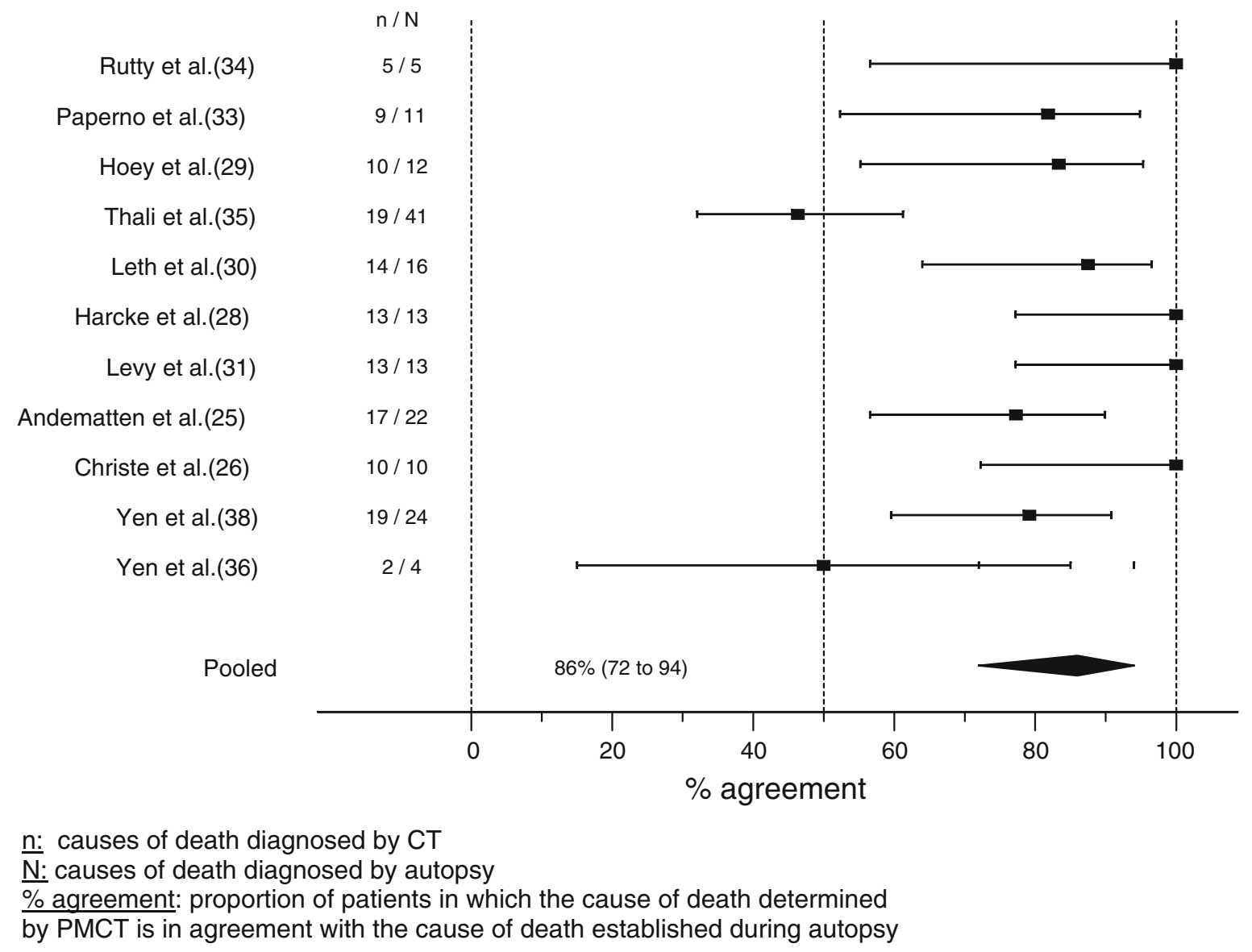

Fig. 2 Forest plot showing percentage agreement on causes of death

Overall, the combination of relatively small studies and substantial differences in design and study population led to substantial heterogeneity in reported percentage agreement (inconsistency $I^{2}=76.4 \%$ ).

The studies included in this systematic review used autopsy as the reference standard and therefore all PMCT findings that were not found during autopsy were considered false positive. More than half of the studies reported significant false positive findings with PMCT [24, $25,27,29,31-33,35,38]$. Some injuries like facial fractures, gas embolisms, small pneumothoraces, pneumopericardium, and pneumomediastinum are difficult to

Table 3 Identified injuries per anatomical region

\begin{tabular}{|c|c|c|c|c|c|c|c|}
\hline Region & $\begin{array}{l}\text { Levy et al. } \\
\text { [31] }\end{array}$ & $\begin{array}{l}\text { Andematten et al. } \\
{[25]}\end{array}$ & $\begin{array}{l}\text { Hoey et al. } \\
\text { [29] }\end{array}$ & $\begin{array}{l}\text { Aghayev et al. } \\
{[24]}\end{array}$ & $\begin{array}{l}\text { Yen et al. } \\
{[36]}\end{array}$ & $\begin{array}{l}\text { Donchin et al. } \\
{[27]}\end{array}$ & $\begin{array}{l}\text { Thali et al. } \\
{[35]}\end{array}$ \\
\hline Head/neck/face & $100(24 / 24)$ & $100(25 / 25)$ & $100(19 / 19)$ & n.a. & $53(28 / 53)$ & n.a. & n.a. \\
\hline Chest & $100(21 / 21)$ & $100(19 / 19)$ & $95(19 / 20)$ & 75-100 (n.a.) & n.a. & n.a. & n.a. \\
\hline Abdomen & $100(3 / 3)$ & $100(1 / 1)$ & $77(10 / 13)$ & n.a. & n.a. & n.a. & n.a. \\
\hline Extremities & $64(18 / 28)$ & $100(1 / 1)$ & n.a. & n.a. & n.a. & n.a. & n.a. \\
\hline External & $100(2 / 2)$ & n.a. & n.a. & n.a. & n.a. & n.a. & n.a. \\
\hline Overall & n.a. & n.a. & n.a. & n.a. & n.a. & $60(57 / 95)$ & $74(48 / 65)$ \\
\hline
\end{tabular}

n.a. not available

Data are presented as \% $(n / N): n$ number of injuries identified by CT, $N$ number of injuries identified by autopsy, $\%$ percentage of identified injuries on PMCT 
detect during conventional autopsy but more easily so with PMCT [29, 35, 38]. To minimize these missed injuries during autopsies some authors advocate specialized maneuvres during the autopsy like "opening the pericardial sac while keeping the organ submerged" or "by using an aspirometer" (techniques to detect gas embolisms) [25, 33]. However, these methods are time consuming and not performed on a routine basis and therefore need frequent practice and education. As a consequence of the difficulty in diagnosing some injuries with autopsy, standard autopsy appears not to be a valid reference standard to test the performance of the PMCT in some cases [27]. To circumvent this problem some authors used the combined findings of PMCT and autopsy as a reference standard [27, 30], resulting in a relatively better performance for PMCT.

Injuries that are most frequently missed by $\mathrm{CT}$ are contusions or superficial lesions of solid organs, small soft tissue contusions, hematoma or emphysema, small brain contusions or hematoma (suggested to be smaller than $3 \mathrm{~mm}$ ), and vessel transections or lacerations. Vessel injuries are often suspected owing to the presence of surrounding hematoma but the specific diagnosis cannot be made. The diagnosis of these injuries might benefit from other modern postmortem imaging techniques such as postmortem angiography [46]. For the detection of small brain contusions or hematomas we suppose that they are better detected on CT examinations with higher resolutions although no hard evidence for this conclusion is found within our review.

Although MRI is known to be more sensitive for distinguishing soft tissue injuries, we did not combine findings from PMCT with MRI because our interest was in the performance of PMCT as a single imaging strategy. Such a single strategy is more relevant for practice as PMCT is less time consuming, more available, cheaper, and easier to interpret than MRI. If MRI is preferred for the diagnosis of specific suspected (soft tissue) injuries it is always possible to perform an additional MRI on indication as suggested by one study [35].

For assessing the performance of PMCT alone we extracted the data specific for PMCT if reported as such in the article. If an article only reported combined findings from CT and MRI, we contacted the authors of these studies if they were able and willing to provide the CT findings only. Five authors were contacted because the data of the PMCT were combined with MRI data. We have subsequently received separate data on MRI and CT findings.

In most studies a standard CT protocol was used. However, the studies were performed between the period of 1994 and 2008, and the system types and the protocols used differed substantially between the studies. Several authors even claim that certain injuries were missed due to the poor resolution [28]. This emphasizes again the need for more standardized CT acquisition and image evaluation protocols in future studies.

In addition, all images were interpreted by radiologists. A drawback could be that not all radiologists are used to interpreting postmortem radiology [32]. Therefore it is possible that findings were not mentioned by the radiologist because they were not familiar with certain postmortem findings [38]. To circumvent this bias, one protocol used both a radiologist and a trainee pathologist for interpretation of the findings on the CT examinations [34] and this was recommended by other authors [29]. Others suggest forensic training for radiologists [24, 38]. Although in this review no hard evidence for beneficial effects could be determined by these adjustments it should logically lead to a better performance of PMCT and we advise this in future study designs.

Finally, because in some studies a relative long period existed between $\mathrm{CT}$ and autopsy (reaching a maximum of 2 days), there is a possibility that some findings developed postmortem. Several authors describe their suspicion concerning such findings [28, 29, 33]. The first study described a distinct subdural hemorrhage detected by autopsy and not shown by CT. The second described intravascular air as a result of postmortem decay. Finally, the third study advocated that the low sensitivity of the PMCT for detecting intracranial hemorrhage and a distinct linear track through the brain after a high-velocity gunshot were a result of decomposition of the brain and subsequent breakdown of blood products. These considerations make it even harder to test the value of PMCT in trauma victims.

Overall, the many differences in study protocols and methods hamper the interpretation of the results. However, despite the fact that this review provides inconsistent evidence for PMCT being a reliable substitute for autopsy in determining the cause of death and specific injuries in trauma victims, in our opinion PMCT can be very useful. Since PMCT in most studies detects large numbers of injuries not seen at autopsy, this imaging method is a good adjunct to autopsy rather that a substitute. Furthermore preautopsy CT can be of guidance to the pathologist during the actual autopsy for specific injuries that are difficult to detect on autopsy alone. Other advantages of PMCT as a postmortem examination are that images can be saved for a long period and data can be studied repeatedly by different specialists. These advantages could potentially be of help in forensic cases [30]. Finally, in this time where autopsy numbers are still declining, PMCT can be an alternative for postmortem examination and an efficient method to obtain epidemiological data and clinical information contributing to optimal patient care. Larger, more standardized, and higher quality studies are needed to further examine the potential role and value of PMCT.

For these future studies we advise that researchers compare the blinded performance of CT with the performance of autopsy. Hereafter, but before closing the body during the autopsy, the CT findings should be revealed to complement the autopsy findings. These combined findings will then be the reference standard. The images should be interpreted by radiologists with forensic experience or assisted by pathologists according to a predefined general 
injury classification. As an injury scoring system we suggest the worldwide accepted AIS for the description of sustained injuries. For the CT protocol we advise the use of high resolution CT images with multiplanar reconstructions. Another method would be to compare the blinded performance of PMCT and autopsy with a third imaging method such as MRI. However, because the value of MRI in postmortem examination is currently also under investigation, this is not the preferred method. Our last recommendation for future studies is a multicenter and international study design to equalize study protocols and increase the study population making subgroup analyses possible and providing high levels of evidence.

\section{Conclusion}

Current studies provide inconsistent evidence as to whether PMCT is a reliable alternative for autopsy in determining the cause of death in trauma victims. PMCT does have several promising features in postmortem examination. As a result PMCT should be used more as an adjunct rather than an alternative to autopsy, because it can diagnose extra injuries which are hard to detect and therefore frequently overseen during conventional autopsies. To examine both the complementary (add-on) and replacement function of PMCT in postmortem examination of trauma victims, there is a need for well-designed and larger prospective studies.

Acknowledgments The authors would like to thank Prof. Dr. K. Yen, Prof. Dr. R.M. Leth, Prof. Dr. Y. Donchin, and Dr. E. Aghayev for their kind replies and additional information on their previously published work.

Conflict of interest TPS is a research fellow at the Trauma Unit Department of Surgery, employed by the AMC Medical Research B. V. and supported by an unrestricted grant from Siemens Medical Solutions, Inc.

Open Access This article is distributed under the terms of the Creative Commons Attribution Noncommercial License which permits any noncommercial use, distribution, and reproduction in any medium, provided the original author(s) and source are credited.

\section{References}

1. Boerma T, Abou-Zahr C, Kinfu Y (2008) World health statistics 2008. http://www.who.int/whosis/whostat/ EN_WHS08 Full.pdf World Health Organization, Geneva. Accessed 23 Nov 2008

2. Mock C, Lormand JD, Goosen J, Joshipura M, Peden M (2004) Guidelines for essential trauma care. World Health Organization, Geneva

3. Baker CC, Oppenheimer L, Stephens B, Lewis FR, Trunkey DD (1980) Epidemiology of trauma deaths. Am J Surg 140:144-150

4. Trunkey DD (1983) Trauma. Accidental and intentional injuries account for more years of life lost in the US than cancer and heart disease. Among the prescribed remedies are improved preventive efforts, speedier surgery and further research. Sci Am 249:28-35

5. Teixeira PG, Inaba K, Hadjizacharia P, Brown C, Salim A, Rhee P, Browder T, Noguchi TT, Demetriades D (2007) Preventable or potentially preventable mortality at a mature trauma center. J Trauma 63:1338-1346

6. Hijar M, Chu LD, Kraus JF (2000) Cross-national comparison of injury mortality: Los Angeles County, California and Mexico City, Mexico. Int J Epidemiol 29:715-721
7. Cothren CC, Moore EE, Hedegaard HB, Meng K (2007) Epidemiology of urban trauma deaths: a comprehensive reassessment 10 years later. World $\mathrm{J}$ Surg 31:1507-1511

8. Soreide K, Kruger AJ, Vardal AL, Ellingsen CL, Soreide E, Lossius HM (2007) Epidemiology and contemporary patterns of trauma deaths: changing place, similar pace, older face. World J Surg 31:2092-2103

9. Yagmur Y, Kiraz M, Kara IH (1999) Looking at trauma and deaths: Diyarbakir city in Turkey. Injury 30:111-114

10. Tien HC, Spencer F, Tremblay LN, Rizoli SB, Brenneman FD (2007) Preventable deaths from hemorrhage at a level I Canadian trauma center. J Trauma 62:142-146

11. Stewart RM, Myers JG, Dent DL, Ermis P, Gray GA, Villarreal R, Blow O, Woods B, McFarland M, Garavaglia J, Root HD, Pruitt BA Jr (2003) Seven hundred fiftythree consecutive deaths in a level I trauma center: the argument for injury prevention. J Trauma 54:66-70

12. Berget E, Ramnefjell M, Svendsen EB, Bertelsen BI, Maehle BO, Svendsen E (2007) [Decline in hospital autopsies.]. Tidsskr Nor Laegeforen 127:2800-2802

13. Fung Kon Jin PH, Klaver JF, Maes A, Ponsen KJ, Das C, Goslings JC (2008) Autopsies following death due to traumatic injuries in The Netherlands: an evaluation of current practice. Injury 39:83-89
14. Loughrey MB, McCluggage WG, Toner PG (2000) The declining autopsy rate and clinicians' attitudes. Ulster Med J 69:83-89

15. O'Grady G (2003) Death of the teaching autopsy. BMJ 327:802-803

16. Ong AW, Cohn SM, Cohn KA, Jaramillo DH, Parbhu R, McKenney MG, Barquist ES, Bell MD (2002) Unexpected findings in trauma patients dying in the intensive care unit: results of 153 consecutive autopsies. J Am Coll Surg 194:401-406

17. Hodgson NF, Stewart TC, Girotti MJ (2000) Autopsies and death certification in deaths due to blunt trauma: what are we missing? Can J Surg 43:130136

18. Bolliger SA, Thali MJ, Ross S, Buck U, Naether S, Vock P (2008) Virtual autopsy using imaging: bridging radiologic and forensic sciences. A review of the Virtopsy and similar projects. Eur Radiol 18:273-282

19. Ljung $\mathrm{P}$, Winskog C, Persson A, Lundstrom C, Ynnerman A (2006) Full body virtual autopsies using a state-ofthe-art volume rendering pipeline. IEEE Trans Vis Comput Graph 12:869876 
20. O'Donnell C, Rotman A, Collett S, Woodford N (2008) Current status of routine post-mortem CT in Melbourne, Australia. Forensic Sci Med Pathol 3:226-232

21. Whiting PF, Weswood ME, Rutjes AW, Reitsma JB, Bossuyt PN, Kleijnen J (2006) Evaluation of QUADAS, a tool for the quality assessment of diagnostic accuracy studies. BMC Med Res Methodol 6:9

22. Brown LD, Cai TT, Dasgupta A (2001) Interval estimation for a binomial proportion. Stat Sci 16:101-133

23. Higgins JP, Thompson SG, Deeks JJ, Altman DG (2003) Measuring inconsistency in meta-analyses. BMJ 327:557-560

24. Aghayev E, Christe A, Sonnenschein M, Yen K, Jackowski C, Thali MJ, Dirnhofer R, Vock P (2008) Postmortem imaging of blunt chest trauma using CT and MRI: comparison with autopsy. J Thorac Imaging 23:20-27

25. Andenmatten MA, Thali MJ, Kneubuehl BP, Oesterhelweg L, Ross S, Spendlove D, Bolliger SA (2008) Gunshot injuries detected by postmortem multislice computed tomography (MSCT): A feasibility study. Leg Med (Tokyo) 10:287-292

26. Christe A, Aghayev E, Jackowski C, Thali MJ, Vock P (2008) Drowningpost-mortem imaging findings by computed tomography. Eur Radiol 18:283-290

27. Donchin Y, Rivkind AI, Bar-Ziv J, Hiss J, Almog J, Drescher M (1994) Utility of postmortem computed tomography in trauma victims. J Trauma 37:552555

28. Harcke HT, Levy AD, Abbott RM, Mallak CT, Getz JM, Champion HR, Pearse L (2007) Autopsy radiography: digital radiographs (DR) vs multidetector computed tomography (MDCT) in high-velocity gunshot-wound victims. Am J Forensic Med Pathol 28:1319
29. Hoey BA, Cipolla J, Grossman MD, McQuay N, Shukla PR, Stawicki SP, Stehly C, Hoff WS (2007) Postmortem computed tomography, "CATopsy", predicts cause of death in trauma patients. J Trauma 63:979-985

30. Leth PM (2008) The use of CT scanning in forensic autopsy. Forensic Sci Med Pathol 3:65-69

31. Levy AD, Abbott RM, Mallak CT, Getz JM, Harcke HT, Champion HR, Pearse LA (2006) Virtual autopsy: preliminary experience in high-velocity gunshot wound victims. Radiology 240:522528

32. Levy G, Goldstein L, Blachar A, Apter S, Barenboim E, Bar-Dayan Y, Shamis A, Atar E (2007) Postmortem computed tomography in victims of military air mishaps: radiological-pathological correlation of CT findings. Isr Med Assoc J 9:699-702

33. Paperno S, Riepert T, Krug B, Rothschild MA, Schultes A, Staak M, Lackner L (2005) [Value of postmortem computed tomography in comparison to autopsy]. Rofo 177:130-136

34. Rutty GN, Robinson CE, BouHaidar R, Jeffery AJ, Morgan B (2007) The role of mobile computed tomography in mass fatality incidents. J Forensic Sci 52:1343-1349

35. Thali MJ, Yen K, Schweitzer W, Vock P, Boesch C, Ozdoba C, Schroth G, Ith $\mathrm{M}$, Sonnenschein M, Doernhoefer T, Scheurer E, Plattner T, Dirnhofer R (2003) Virtopsy, a new imaging horizon in forensic pathology: virtual autopsy by postmortem multislice computed tomography (MSCT) and magnetic resonance imaging (MRI) - a feasibility study. J Forensic Sci 48:386-403

36. Yen K, Thali MJ, Aghayev E, Jackowski C, Schweitzer W, Boesch C, Vock P, Dirnhofer R, Sonnenschein M (2005) Strangulation signs: initial correlation of MRI, MSCT, and forensic neck findings. J Magn Reson Imaging 22:501-510

37. Yen K, Sonnenschein M, Thali MJ, Ozdoba C, Weis J, Zwygart K, Aghayev E, Jackowski C, Dirnhofer R (2005) Postmortem multislice computed tomography and magnetic resonance imaging of odontoid fractures, atlantoaxial distractions and ascending medullary edema. Int $\mathrm{J}$ Legal Med 119:129-136
38. Yen K, Lovblad KO, Scheurer E, Ozdoba C, Thali MJ, Aghayev E, Jackowski C, Anon J, Frickey N, Zwygart K, Weis J, Dirnhofer R (2007) Post-mortem forensic neuroimaging: correlation of MSCT and MRI findings with autopsy results. Forensic Sci Int 173:21-35

39. Hayakawa M, Yamamoto S, Motani H, Yajima D, Sato Y, Iwase H (2006) Does imaging technology overcome problems of conventional postmortem examination? A trial of computed tomography imaging for postmortem examination. Int J Legal Med 120:24-26

40. Levy AD, Harcke HT, Getz JM, Mallak CT, Caruso JL, Pearse L, Frazier AA, Galvin JR (2007) Virtual autopsy: twoand three-dimensional multidetector $\mathrm{CT}$ findings in drowning with autopsy comparison. Radiology 243:862-868

41. Poulsen K, Simonsen J (2007) Computed tomography as routine in connection with medico-legal autopsies. Forensic Sci Int 171:190-197

42. Yamazaki K, Shiotani S, Ohashi N, Doi M, Kikuchi K, Nagata C, Honda K (2006) Comparison between computed tomography (CT) and autopsy findings in cases of abdominal injury and disease. Forensic Sci Int 162:163-166

43. Landis JR, Koch GG (1977) The measurement of observer agreement for categorical data. Biometrics 33:159-174

44. Moher D, Cook DJ, Eastwood S, Olkin I, Rennie D, Stroup DF (1999) Improving the quality of reports of metaanalyses of randomised controlled trials: the QUOROM statement. Quality of reporting of meta-analyses. Lancet 354:1896-1900

45. AAAM Committee on Injury Scaling (1998) Abbreviated injury scale (AIS) (1990 revision) - update 98. Association for the Advancement of Automotive Medicin, Des Plains, IL

46. Jackowski C, Sonnenschein M, Thali MJ, Aghayev E, von AG, Yen K, Dirnhofer R, Vock P (2005) Virtopsy: postmortem minimally invasive angiography using cross section techniques - implementation and preliminary results. J Forensic Sci 50:1175-1186 